\title{
Atomic force microscopy study of laser induced phase transitions in $\mathrm{Ge}_{2} \mathrm{Sb}_{2} \mathrm{Te}_{5}$
}

V. Weidenhof, I. Friedrich, S. Ziegler, and M. Wuttig

Citation: Journal of Applied Physics 86, 5879 (1999);

View online: https://doi.org/10.1063/1.371606

View Table of Contents: http://aip.scitation.org/toc/jap/86/10

Published by the American Institute of Physics

\section{Articles you may be interested in}

Structural transformations of $\mathrm{Ge}_{2} \mathrm{Sb}_{2} \mathrm{Te}_{5}$ films studied by electrical resistance measurements Journal of Applied Physics 87, 4130 (2000); 10.1063/1.373041

Laser induced crystallization of amorphous $\mathrm{Ge}_{2} \mathrm{Sb}_{2} \mathrm{Te}_{5}$ films Journal of Applied Physics 89, 3168 (2001); 10.1063/1.1351868

Rapid-phase transitions of $\mathrm{GeTe}_{\mathrm{S}} \mathrm{Sb}_{2} \mathrm{Te}_{3}$ pseudobinary amorphous thin films for an optical disk memory Journal of Applied Physics 69, 2849 (1998); 10.1063/1.348620

Nanosecond switching in GeTe phase change memory cells Applied Physics Letters 95, 043108 (2009); 10.1063/1.3191670

Investigation of the optical and electronic properties of $\mathrm{Ge}_{2} \mathrm{Sb}_{2} \mathrm{Te}_{5}$ phase change material in its amorphous, cubic, and hexagonal phases

Journal of Applied Physics 97, 093509 (2005); 10.1063/1.1884248

Density changes upon crystallization of $\mathrm{Ge}_{2} \mathrm{Sb}_{2.04} \mathrm{Te}_{4.74}$ films

Journal of Vacuum Science \& Technology A: Vacuum, Surfaces, and Films 20, 230 (2002); 10.1116/1.1430249

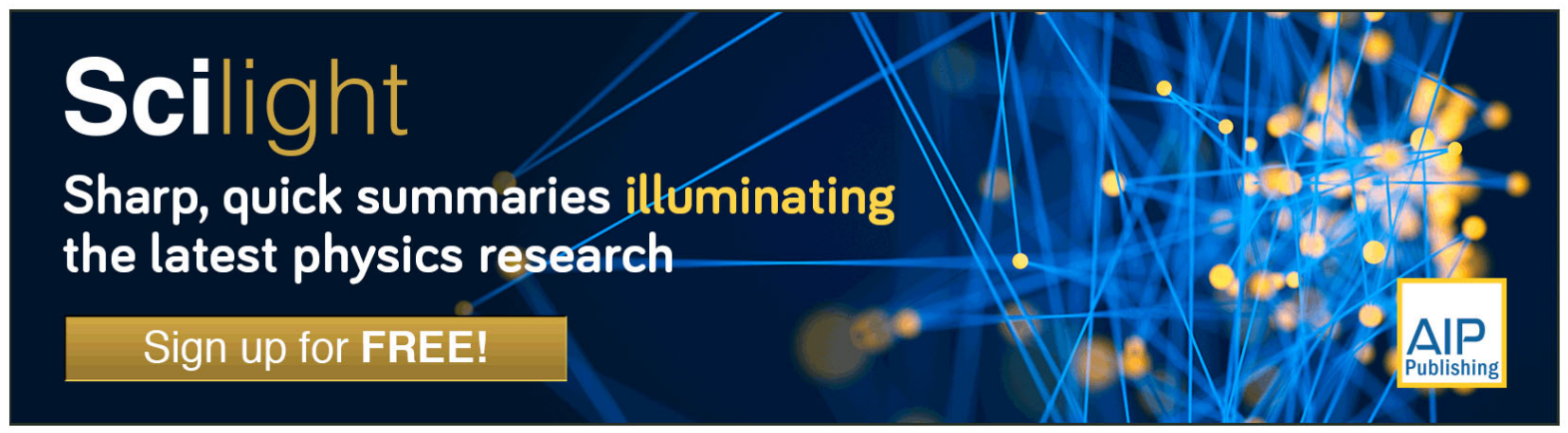




\title{
Atomic force microscopy study of laser induced phase transitions in $\mathrm{Ge}_{2} \mathrm{Sb}_{2} \mathrm{Te}_{5}$
}

\author{
V. Weidenhof ${ }^{\text {a) }}$ and I. Friedrich \\ Institut für Grenzflächenforschung und Vakuumphysik, Forschungszentrum Jülich, D-52425 Jülich, Germany \\ and I. Physikalisches Institut der RWTH Aachen, D-52056 Aachen, Germany \\ S. Ziegler \\ Institut für Grenzflächenforschung und Vakuumphysik, Forschungszentrum Jülich, D-52425 Jülich, Germany \\ M. Wuttig \\ Institut für Grenzfächenforschung und Vakuumphysik, Forschungszentrum Jülich, D-52425 Jülich, Germany \\ and I. Physikalisches Institut der RWTH Aachen, D-52056 Aachen, Germany
}

(Received 25 May 1999; accepted for publication 10 August 1999)

\begin{abstract}
Micron- and submicron-size changes induced by local laser heating in thin films of $\mathrm{Ge}_{2} \mathrm{Sb}_{2} \mathrm{Te}_{5}$ are studied by atomic force microscopy (AFM). This material is presently used for rewritable data storage that employs phase change recording. Reversible switching between the amorphous and crystalline states, which is accompanied by a considerable change in optical properties and film density, is exploited to store bits of information. The density change can be detected by AFM, which we use here to study the amorphization (writing) and recrystallization (erasure) of single bits. Both processes have been measured as a function of modification pulse power and duration. Morphology changes can be detected even if the phase change film is covered by a thin protective layer. AFM allows a precise determination of the bit size and bit depth, which characterizes the progress of the phase change in the direction of the surface normal. The present setup allows the correlation of the change in optical reflectance $\Delta R$ caused by a specific laser pulse to the bit topography. This enables a detailed study of the mechanisms of bit writing and erasure. (C) 1999 American Institute of Physics. [S0021-8979(99)00222-4]
\end{abstract}

\section{INTRODUCTION}

Rewritable optical data storage play a key role for future multimedia applications. Phase change recording is a particularly promising technique for future optical data storage since it is conceptionally compatible with present $\mathrm{CD}$ and $\mathrm{CD}-\mathrm{R}$ formats. ${ }^{1-5}$ The principle behind phase change recording is the reversible transformation of small areas (bits) of the active layer between the stable crystalline and the metastable amorphous phases. ${ }^{6}$ Applying a short pulse of a focused laser beam to a crystalline area increases the local temperature above the melting point. When the pulse ends, the molten spot cools rapidly and amorphizes. The amorphous and crystalline phases have very different optical properties. Therefore the data can be read by monitoring the local changes in the reflection or transmission of the media. Erasure is achieved by laser heating to intermediate temperatures, which enables the fast recrystallization of amorphous bits. This reversible transformation allows rewritable data storage. GeSbTe alloys are particularly well suited for satisfying all the requirements associated with data storage applications. ${ }^{7}$ The pronounced difference in the optical properties leads to high contrast, which is one of the attractive features of phase change recording.

Nevertheless this technology needs further development to ensure its future success. Of paramount importance is the improvement of data transfer rates, i.e., the time required to

${ }^{a)}$ Electronic mail: v.weidenhof@fz-juelich.de write and erase bits, and the storage density, i.e., the area occupied by a single bit. This implies that novel concepts for optical data storage such as near-field recording need to be pursued..$^{8-12}$ With bit sizes considerably below the $\mu \mathrm{m}$ mark, techniques which allow the microscopy of single bits on the nanometer scale become increasingly important.

In this article we will demonstrate that atomic force microscopy (AFM) can be used as a versatile tool to monitor the bit topography. In contrast to transmission electron microscopy there is no need for time consuming sample preparation. AFM provides a three-dimensional image of the changes in film morphology due to the phase change. Therefore AFM can monitor the progress of the phase change as a function of the laser power and/or pulse length applied. We will show that the bit topography is directly related to the reflectance change measured. This establishes the use of the analytical power of AFM for dedicated efforts in improving the performance, e.g., data transfer rate and signal-to-noise ratio, as well as the storage density of phase change media.

\section{EXPERIMENTAL DETAILS}

Thin films of $\mathrm{Ge}_{2} \mathrm{Sb}_{2} \mathrm{Te}_{5}$ were deposited at room temperature on glass substrates or $\mathrm{Si}$ wafers by dc-magnetron sputtering using an alloy target $(10 \mathrm{~cm}$ in diameter) with the above composition. The background pressure of the sputter system was $2 \times 10^{-7}$ mbar. Typically an Ar pressure of 5 $\times 10^{-3}$ mbar and $100 \mathrm{~W}$ applied power were used to deposit the films. The resulting growth rate was $5 \mathrm{~nm} / \mathrm{s}$. X-ray dif- 
fraction measurements confirmed that the as deposited $\mathrm{Ge}_{2} \mathrm{Sb}_{2} \mathrm{Te}_{5}$ films were amorphous. Some of the samples were crystallized in a furnace under a protective Ar atmosphere. During the annealing procedure the amorphous film transforms into either a face-centered-cubic (fcc) or a hexagonal phase depending on the annealing parameters. ${ }^{13}$ Optical spectroscopy shows that the hexagonal phase exhibits slightly different optical properties from that of the fcc phase. ${ }^{14}$ The resulting difference in reflectance between the fcc and hexagonal phases depends on the film thickness and is $\sim 10 \%$ for the films investigated in this study.

A static tester was built to modify the active layer and to monitor the changes in optical properties upon writing and erasing bits. In our setup, a pulsed GaAs laser diode with a wavelength of $830 \mathrm{~nm}$ was used. Pulse powers up to $38 \mathrm{~mW}$ and pulse durations as short as $3 \mathrm{~ns}$ can be employed. The collimated laser beam has a Gaussian profile with a diameter of $3 \mathrm{~mm}$. It is focused onto the film by a microscope objective with a numerical aperture (NA) of 0.9 or 0.4 , respectively. A low power probe pulse was used to measure the reflectance before and after either crystallization or amorphization pulses. The relative change in reflectance $\Delta R$ was recorded as

$$
\Delta R=\frac{R_{c}-R_{a}}{R_{c}},
$$

where $R_{c}$ and $R_{a}$ are the reflectances of the crystalline and amorphous phases, respectively. The recorded bits were examined by atomic force microscopy. The Dimension 3100 AFM from Digital Instruments that was used is equipped with a video microscope. This enables identification of suitably marked regions. As a consequence, individual bits which have caused a well known change in reflectance $\Delta R$ can be selectively imaged. Hence we can unequivocally correlate a change in reflectance to the bit topography measured by the AFM.

\section{RESULTS}

\section{A. Crystalline bits in an amorphous matrix}

Figure 1 shows an AFM image of crystalline areas in an amorphous film on a glass substrate. The film thickness is $\sim 80 \mathrm{~nm}$. The amorphous film exhibits a very small root mean square (rms) roughness of typically $3 \AA$. The incident laser power was 3.6 [Fig. 1(a)] and $2.1 \mathrm{~mW}$ [Fig. 1(b)]. The laser pulse length was $100 \mathrm{~ns}$ [Fig. 1(a)] and $5 \mathrm{~ms}$ [Fig. 1(b)], respectively. The laser beam was focused by an objective lens with $\mathrm{NA}=0.9$. Crystallization leads to an increase in film density. Therefore the crystallized region corresponds to a depression in the amorphous surrounding. The mean depth $\bar{d}$ of the bits is 2.5 [Fig. 1(a)] and $2.8 \mathrm{~nm}$ [Fig. 1(b)], respectively. The modified area has a circular shape with radius of 500 [Fig. 1(a)] or $400 \mathrm{~nm}$ [Fig. 1(b)] and reveals sharp edges. The structure inside the bit shown in Fig. 1(a) is not homogeneous but it reveals several microcracks. The measured maximum depth of the cracks is $6 \mathrm{~nm}$ but the actual depth could be considerably larger since it is difficult for the AFM tip to image such narrow cracks. A much more homo-
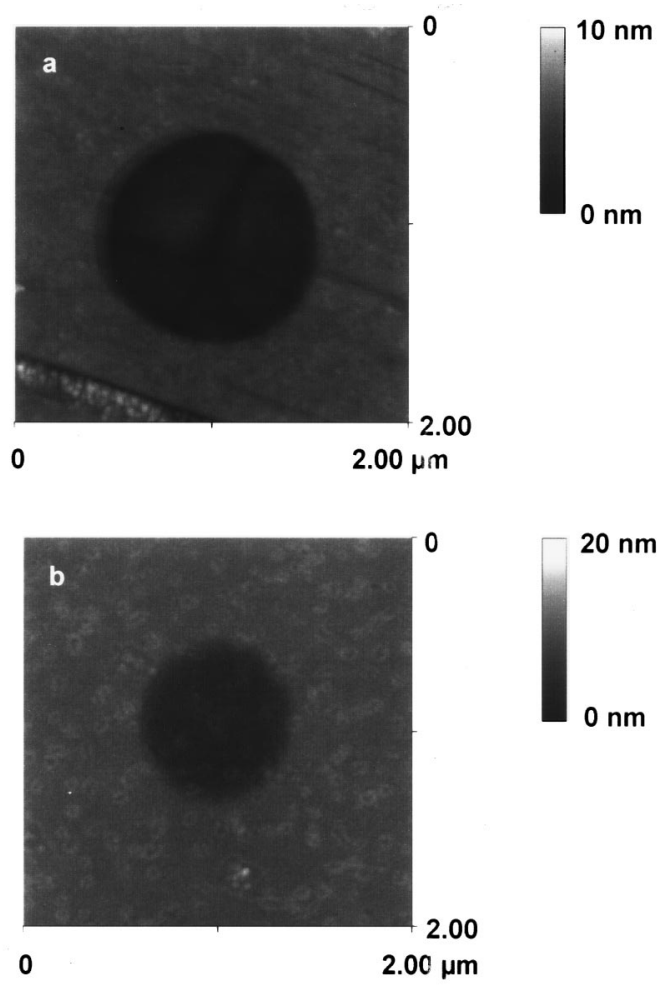

FIG. 1. AFM micrograph of crystalline bits in an $80 \mathrm{~nm}$ thick amorphous film on a glass substrate. The incident laser power, is 3.6 (a) and (b) 2.1 $\mathrm{mW}$, respectively. The pulse duration is (a) $100 \mathrm{~ns}$ and (b) $5 \mathrm{~ms}$, respectively. The short crystallization pulse leads to the formation of microcracks.

geneous bit structure was obtained using longer pulses [see Fig. 1(b)]. In this case the AFM micrograph reveals a smooth bit topography without any cracks.

A characteristic parameter of the bits is the volume reduction, i.e., the volume decrease due to crystallization. Figure 2(a) shows the reduction of volume as a function of pulse length. The laser beam was focused by an objective lens with $\mathrm{NA}=0.4$. One can clearly distinguish three phases. In phase I $(t<40 \mathrm{~ns})$, there is no measurable change in volume. During phase II ( $40 \mathrm{~ns}<t<400 \mathrm{~ns})$, there is a steep increase in the volume reduction, followed by a weak increase in phase III ( $t>400 \mathrm{~ns})$. By decomposing the volume reduction into the area and mean depth of the depression (bit) one can see that the pronounced increase of volume reduction in phase II is due to simultaneous growth of the area and the depth of the bit [Figs. 2(b) and 2(c)]. The depth is constant in phase III and the volume increase is only due to an increase in the bit area. The saturation depth is $5.1 \pm 0.3 \mathrm{~nm}$. The thickness of the as deposited amorphous film is $85 \pm 3 \mathrm{~nm}$. This leads to a change in density of $6.0 \pm 0.6 \%$.

The same change in density can also be monitored by $\mathrm{X}$-ray-reflectometry (XRR) for samples modified on a macroscopic scale by annealing them in an oven under a protective Ar atmosphere. XRR allows one to determine the density of thin films by measuring the angular dependence of the specularly reflected intensity at small incidence angles. ${ }^{15,16}$ The position of the total reflection edge reveals the film density. An amorphous film obtained after sputtering was analyzed first. The XRR measurements of this sample determine 

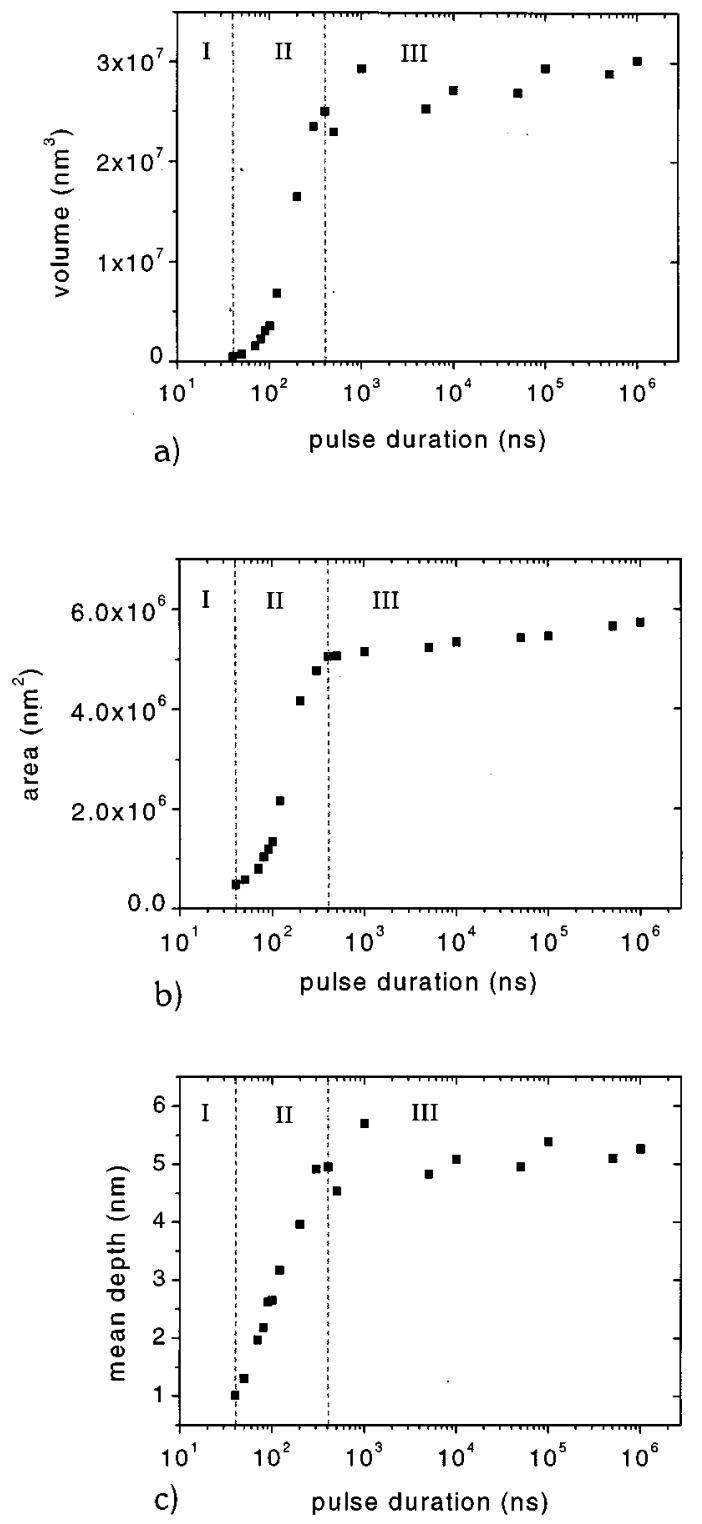

FIG. 2. (a) Volume reduction, (b) bit area, and (c) mean depth $\bar{d}$ of crystalline bits as a function of pulse duration. The sample was an $80 \mathrm{~nm}$ thick amorphous $\mathrm{Ge}_{2} \mathrm{Sb}_{2} \mathrm{Te}_{5}$ film on a Si wafer. The development of the bit can be divided in three phases.

the density to $6.00 \pm 0.03 \mathrm{~g} / \mathrm{cm}^{3}$. Then the same sample was annealed in a furnace for $30-60 \mathrm{~min}$ at $170^{\circ} \mathrm{C}$ in order to obtain the crystalline fcc structure and then was subsequently analyzed again. After annealing the density increases to 6.4 $\pm 0.1 \mathrm{~g} / \mathrm{cm}^{3}$. This leads to a change in density of 6.2 $\pm 0.8 \%$. A possible further increase in density due to the transformation from the fcc to the hexagonal phase is of the same order of magnitude as the error of $0.1 \mathrm{~g} / \mathrm{cm}^{3}$. The value of $6.2 \pm 0.8 \%$ determined by XRR is in good agreement with the AFM measurements which show a $6.0 \pm 0.6 \%$ volume reduction. Hence it can be concluded that the saturation in bit depth measured by AFM occurs once crystallization in the direction normal to the sample surface is complete. Therefore the mean depth of the bit can be used as a measure of the degree of crystallization inside the film.
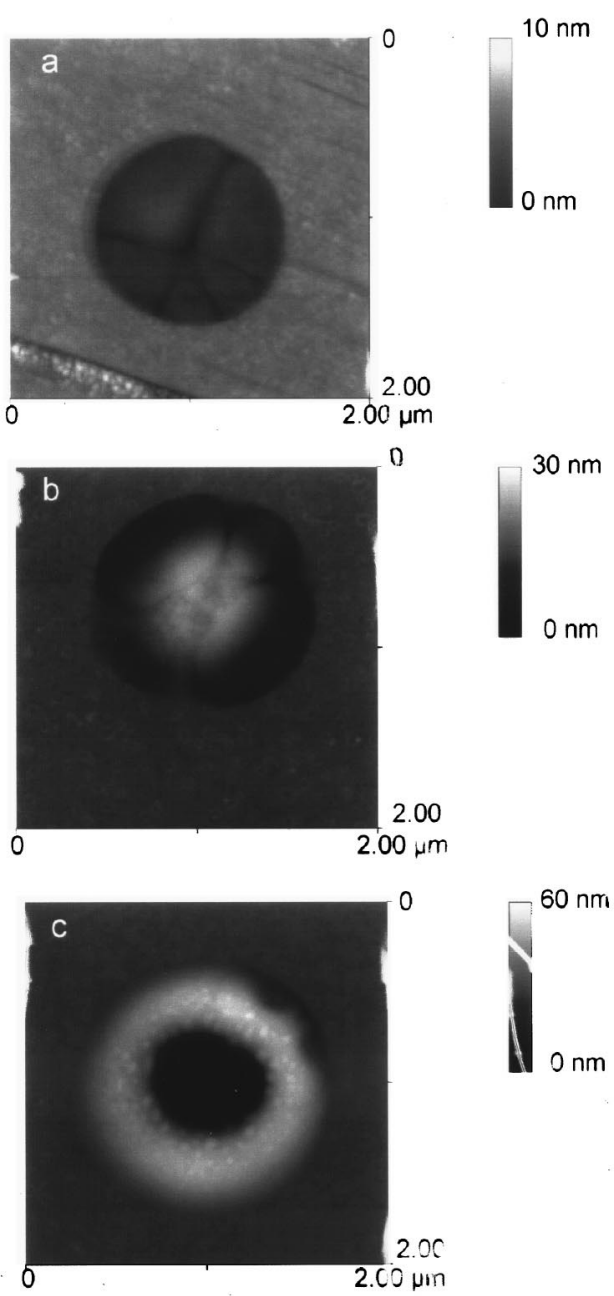

FIG. 3. (a) Crystallization, (b) reamorphization, and finally (c) ablation observed upon increasing laser power.

The temperature of the locally heated region increases linearly with the applied laser power. ${ }^{17}$ By increasing the laser power, the central region of the bit will reach the melting temperature. Then for short laser pulses reamorphization of the inner part of a bit can occur due to melting followed by quenching. An example of this is shown in Figs. 3(a) and 3(b). The bit in Fig. 3(a) is created by a pulse of $3.6 \mathrm{~mW}$ for 100 ns. A typical crystallization pattern emerges. Increasing the laser power and hence the temperature leads to reamorphization as is shown in Fig. 3(b). Here a $7.0 \mathrm{~mW}, 100 \mathrm{~ns}$ pulse leads to the formation of a $8 \mathrm{~nm}$ high hillock inside a 3 $\mathrm{nm}$ deep crystalline bit. The amorphous phase has a lower density than the crystalline phase. Therefore the reamorphized region appears as a hillock in the middle of the crystalline area. A further increase in laser power finally leads to ablation, which is characterized by a deep crater surrounded by a mound. An example of this is shown in Fig. 3(c). The mound is $30 \mathrm{~nm}$ high. Please note that the bottom of the crater is $78 \mathrm{~nm}$ below the film surface. This value corresponds to the film thickness. Hence the crater formation is due to the complete removal of the film.

Our experimental setup allows correlation between the change in the optical signal $\Delta R$ upon bit generation on the one hand and the topological data of the same bit as mea- 

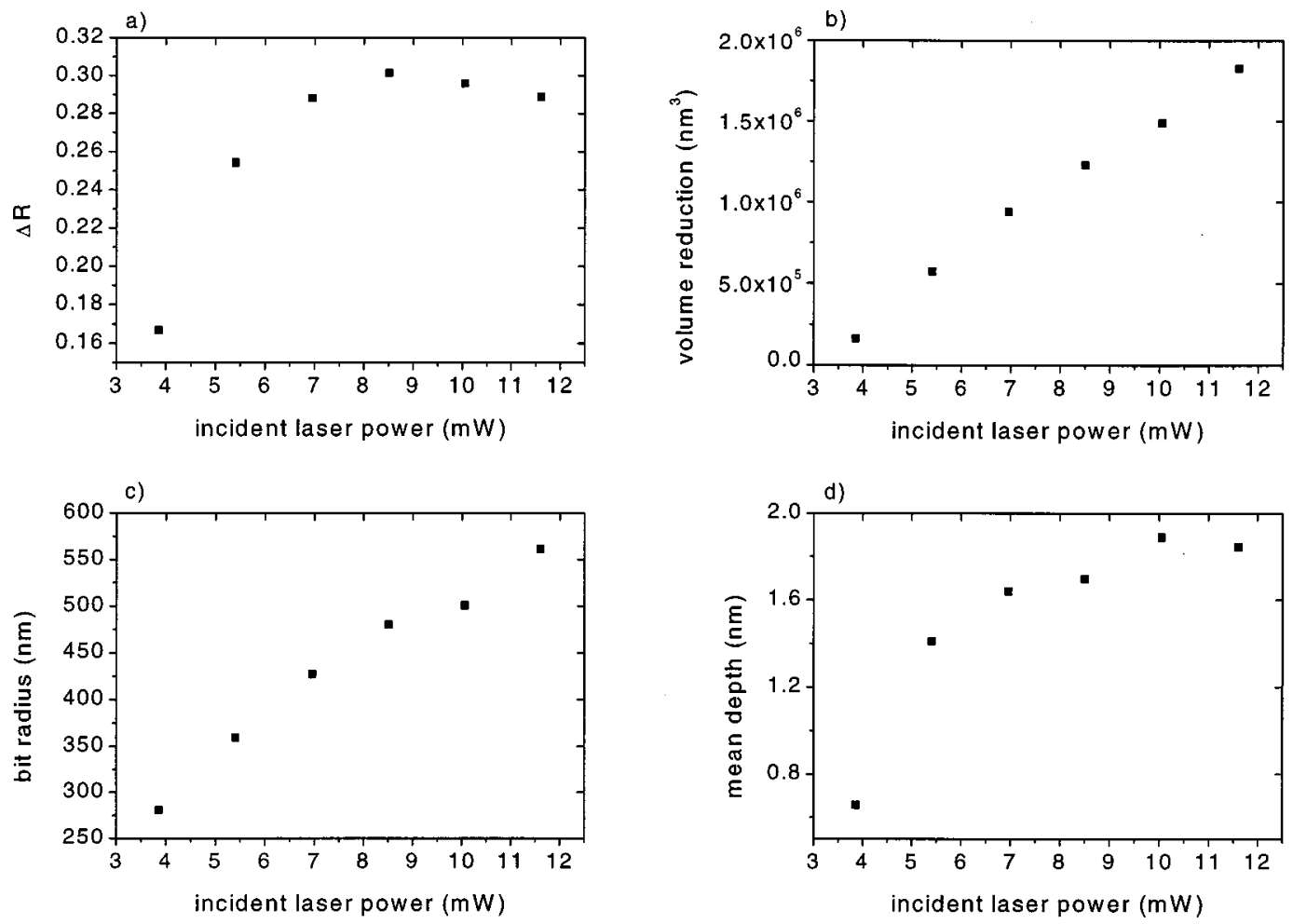

FIG. 4. (a) Development of reflectance change $\Delta R$, (b) volume reduction, (c) radius, and (d) mean depth of crystalline bits in an amorphous film on a Si wafer as a function of applied laser power. A comparison of (a) and (d) reveals a strong correlation between the mean depth of the bit and changes in the optical reflectance.

sured by AFM on the other. As mentioned above, we are able to identify individual bits in the AFM and therefore can examine bits which have caused a specific reflectance change $\Delta R$. Figure 4(a) shows the development of $\Delta R$ with laser pulse power for a series of six crystalline bits in an amorphous matrix on a Si substrate. The pulse duration was $1 \mu \mathrm{s}$ and the applied power varied from 3.9 to $11.9 \mathrm{~mW}$. The laser beam was focused by an objective lens with a NA of 0.9. At first glance, the change in reflectance $\Delta R$ shows hardly any correlation with the volume reduction of the bits [Fig. 4(b)]. But decomposing the volume into the bit radius [Fig. 4(c)] and mean depth $\bar{d}$ [Fig. 4(d)] shows that a strong correlation exists between $\Delta R$ and $\bar{d}$, whereas no significant dependence of $\Delta R$ upon the bit radius is found. The latter can be explained by the high numerical aperture of the objective lens. It leads to a focus spot with a Gaussian radius of approximately $300 \mathrm{~nm}$. Therefore an increase in bit radius beyond $300 \mathrm{~nm}$ should not lead to a significant increase in $\Delta R$. On the contrary, an increase in the mean depth of the crystallized region leads to an increase in $\Delta R$ since $\bar{d}$ is a measure of the degree of crystallization inside the film. Such a finding is expected as long as the crystallized layer is not completely opaque. For a completely opaque layer, a further increase in the degree of crystallization will not influence the reflectance. The correlation between $\Delta R$ and $\bar{d}$ specified above is expected to hold for crystalline layers less than $90 \mathrm{~nm}$ thick. In the case presented in Fig. $4, \Delta R$ depends only on $\bar{d}$. In general, however, the reflectance change $\Delta R$ will depend both on the radius and on the depth of the crystallized bit. Hence optical measurements alone are insufficient to deter- mine the microscopic structure of the bits from reflectance data. The AFM on the contrary can be used to unambiguously relate a change of $\Delta R$ to bit size and bit depth.

\section{B. Amorphous bits in a crystalline matrix}

In Sec. III A, crystalline bits in an amorphous matrix were discussed. In the present implementation of phase change recording, amorphous bits are written into a crystalline film. Therefore amorphous bits in a crystalline matrix were also examined. Crystalline samples were prepared by furnace annealing of as deposited amorphous films. The rms roughness of the sample surface typically increases upon annealing up to $10 \AA$ as was confirmed by the AFM. Crystalline films with both fcc and hexagonal structures were investigated. Amorphous bits in a fcc matrix are presented first.

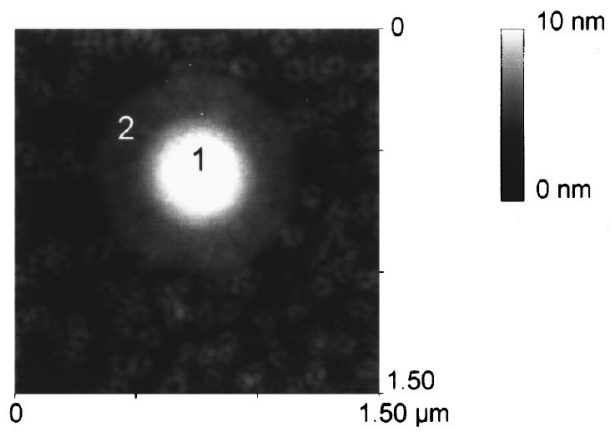

FIG. 5. Amorphous bit in a fcc surrounding. The pulse parameters were $23.2 \mathrm{~mW}$ and $89 \mathrm{~ns}$. The bit consists of two zones, "1" and " 2 ," respectively. 

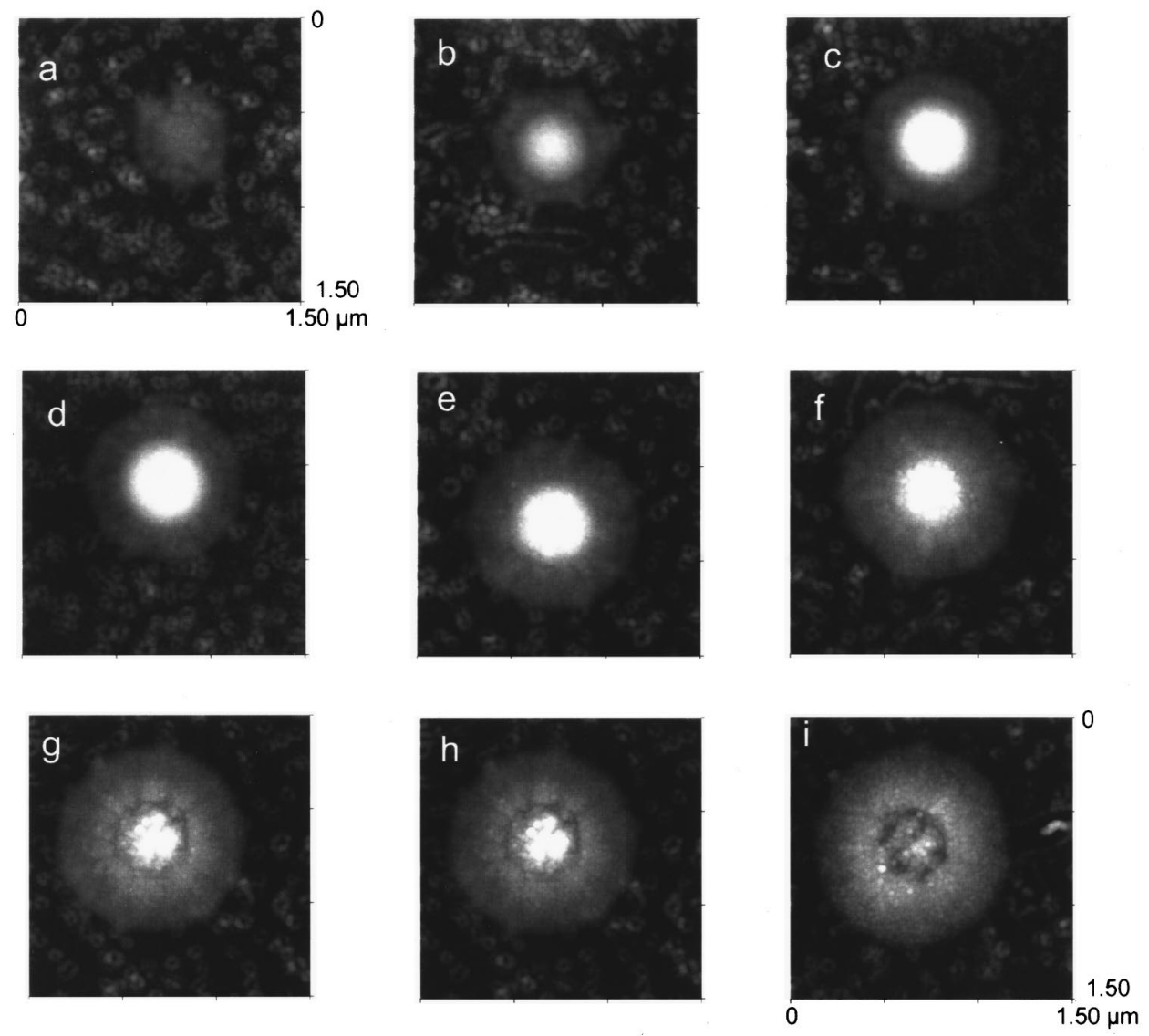

FIG. 6. (a)-(i) Series of AFM images of amorphous bits. The applied pulse power was $23.2 \mathrm{~mW}$. The pulse duration varied from 30 to $266 \mathrm{~ns}$. Optical characterization of the bits is shown in (j). (j) Change in reflectance $\Delta R$ as a function of pulse length. Some of the bits are shown in (a)-(d). The characters displayed near the appropriate data points identify the corresponding AFM image.

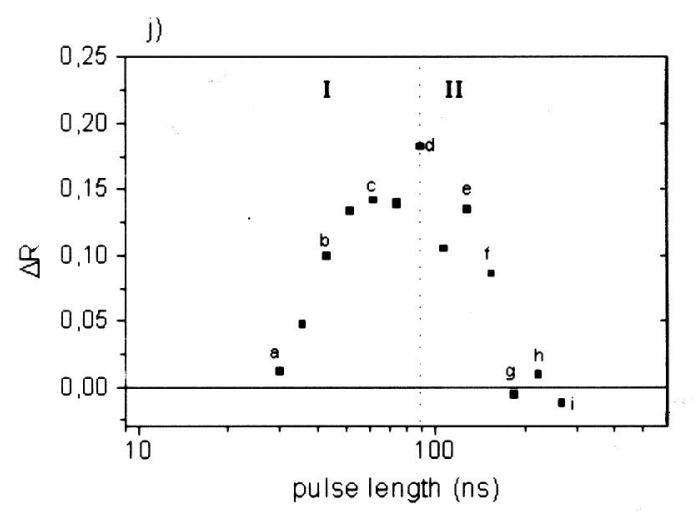

A typical AFM micrograph of an amorphous bit in a crystalline film with a fcc structure is shown in Fig. 5. The film thickness is $\sim 75 \mathrm{~nm}$. Besides a decrease in reflectance, amorphization leads also to a decrease in density and therefore to a local increase in film thickness. The diameter of the bit in Fig. 5 is $860 \mathrm{~nm}$ and the height is $8 \mathrm{~nm}$. Amorphous bits typically consists of two zones, labeled " 1 "' and " 2 "' in Fig. 5. There is a center mound (1) surrounded by a lower corona (2). In order to clarify if the whole structure or just the center mound consists of amorphous material, a series of amorphous bits was examined both optically and by AFM. The incident laser power was held constant and the pulse length $t_{a}$ varied from 25 to 266 ns. Figures 6(a)-6(i) show AFM images of some of the characterized bits. In a first stage [Figs. 6(a)-6(d)] there is an increase of the center mound. In a subsequent second stage [Figs. 6(e)-6(i)] a decrease of the mound can be observed. Both in the first and second stages an increase of the corona can be seen.

The corresponding changes in reflectance $\Delta R$ evoked by the same bits are plotted in Fig. 6(j) although the amorphization leads to a decrease in reflectance, $\Delta R$ is plotted as a positive variable since the calculation of $\Delta R$, defined in Eq. (1), always leads to positive values. The optical data also show two stages, labeled I and II in Fig. 6(j). For pulses as short as $90 \mathrm{~ns}$ or less [bits (a)-(d), stage I] there is an increase in $\Delta R$ with increasing $t_{a}$. This is expected since the amount of molten and subsequently quenched material increases. Thus the amorphized fraction and also $\Delta R$ increase. But in stage II [bits (e)-(i)] $\Delta R$ decreases with $t_{a}$ and finally reaches zero. This can be explained by a cooling rate which has become too low for efficient quenching of the melt in stage II. The decrease in the cooling rate for long pulses stems from mainly two reasons. On the one hand, an increase in the amount of molten material means a decrease in the 

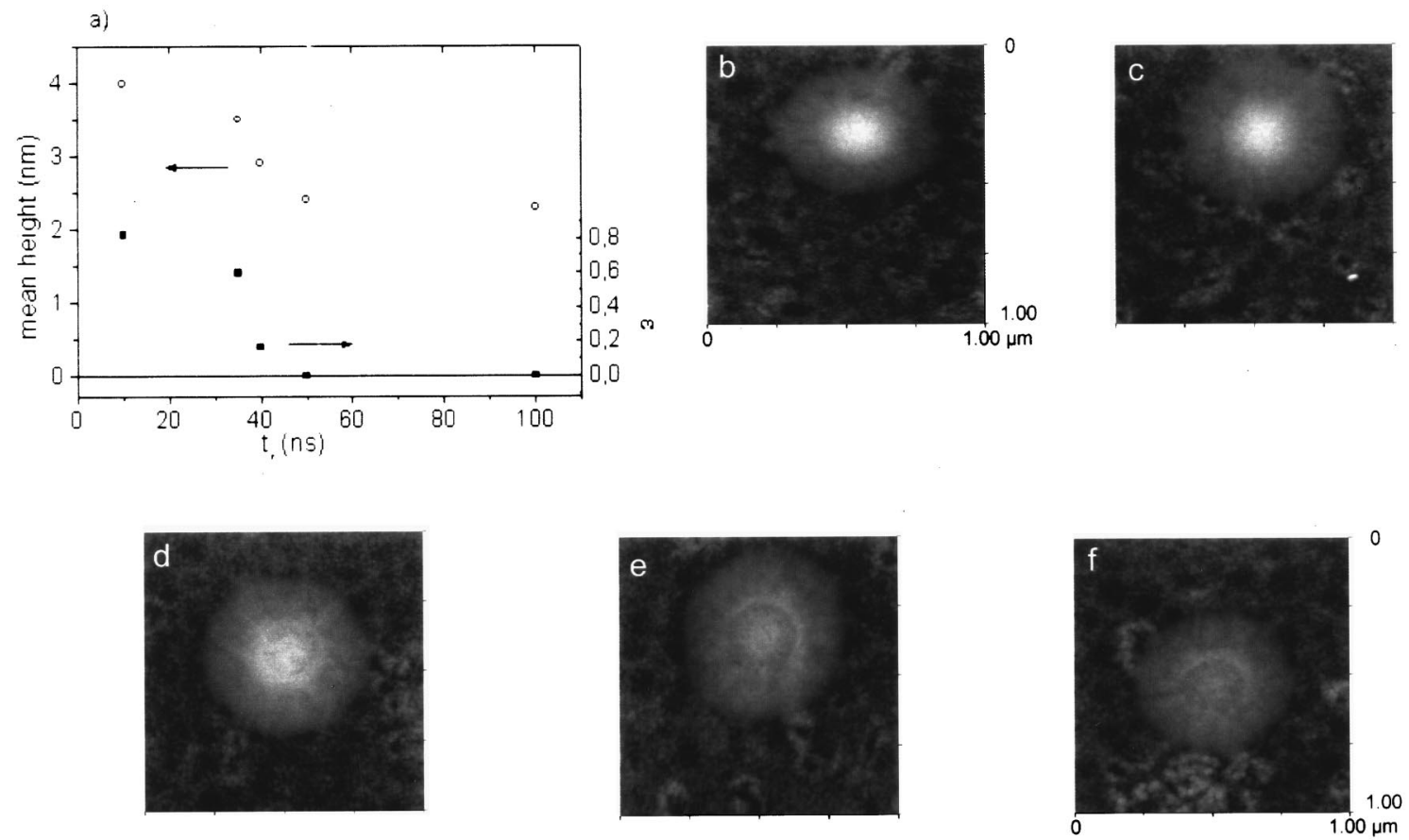

FIG. 7. (a) Remaining amorphous fraction $\epsilon$ for various partially and completely erased bits in fcc film as a function of the duration of the recrystallization pulse (closed squares). After $50 \mathrm{~ns}$ the reflectance reaches its original value $(\epsilon=0)$, and complete erasure is achieved. The mean height of the same bits after the recrystallization pulse is also plotted (open circles). (b)-(f) A series of partially and completely erased (recrystallized) bits. The amorphization parameters were $7.4 \mathrm{~mW}$ and $100 \mathrm{~ns}$. The power of the recrystallization pulse was $2.4 \mathrm{~mW}$. The duration of the pulse was $10,30,35,40$, and $100 \mathrm{~ns}$, respectively. Recrystallization leads to a decrease in bit height, whereas the diameter of the bits remains unchanged. Nevertheless, even the completely erased bits are still visible by AFM in (f).

ratio between the surface and the volume of the melt. On the other hand, long pulses lead to increased heating of the area surrounding the melt and thus to a smaller temperature gradient. As a consequence, the cooling rate decreases and it is in stage II, so slow that quenching becomes more and more difficult. Therefore an increasing fraction of the melt is able to transform into the favorable crystalline state. Hence $\Delta R$ decreases and reaches finally zero when all the molten material recrystallizes upon cooling. One can clearly see that an increase in $\Delta R$ corresponds to an increase of the center mound [Figs. 6(a) $-6(\mathrm{~d})$, stage I], while a decrease in $\Delta R$ corresponds to a decrease of the central mound [Figs. 6(e)6(i), stage II]. In contrast, the coronal region increases in stage I as well as in stage II. Hence it can be concluded that only the center mound consists of amorphous material. Since $\Delta R$ reaches zero, the optical properties of the coronal region correspond to those of the crystalline fcc phase.

Here in Sec. III B the erasure of amorphous bits in a fcc surrounding will be examined. Erasure corresponds to the recrystallization of bits. This is achieved by applying a laser pulse of appropriate power and length. The amorphization of the bits that will be shown here was performed with an incident laser power of $7.8 \mathrm{~mW}$. The length of the amorphization pulse was $100 \mathrm{~ns}$. The power of the recrystallization pulse was held constant at $2.4 \mathrm{~mW}$ and the pulse duration was varied. The laser beam was focused by an objective lens with a NA of 0.9. The erasure process is studied with both AFM and optical measurements. After application of the recrystallization pulse the reflectance increases and reaches the original value when the amorphous bit is completely recrystal- lized. Partial recrystallization leads to reflectance that is less than the original value. The reflectance was measured before the amorphization pulse $\left(R_{c}\right)$, after the amorphization pulse $\left(R_{a}\right)$ and finally after the recrystallization pulse $\left(R_{r}\right)$. This allows one to determine the remaining amorphous fraction $\epsilon$ as a function of the duration $t_{r}$ of the recrystallization pulse according to the following equation:

$$
\epsilon=\frac{R_{c}-R_{r}}{R_{c}-R_{a}} ;
$$

$\epsilon=1$ is obtained for $R_{r}=R_{a}$, i.e., no recrystallization. $\epsilon$ $=0$ is obtained for $R_{r}=R_{c}$, i.e., complete recrystallization of the amorphous mark. The corresponding data are plotted in Fig. 7(a) (shown by closed squares). $t_{r}$ varies from 10 to $100 \mathrm{~ns}$. One can see clearly that the $50 \mathrm{~ns}$ pulse is sufficient for complete recrystallization $(\epsilon=0)$ while the $10 \mathrm{~ns}$ pulse shows only a small effect $(\epsilon=0.82)$. The strongest change in reflectance occurs between 35 and 50 ns. Figures 7(b)-7(f) show AFM images of the partially erased amorphous bits characterized optically in Fig. 7(a). After the 10 ns recrystallization pulse a pronounced center mound is still visible [Fig. 7(b)]. The height of the mound decreases for longer recrystallization pulses but the coronal region around the mound remains unchanged even if the bits are completely erased from the optical point of view [Fig. 7(f)]. The reflectance change is closely correlated with the mean height $\bar{h}$ of the partly recrystallized bits [shown by open circles in Fig. 7(a)]. A reduction in $\bar{h}$ is accompanied by a reflectance change. Nevertheless there is one obvious difference; while the re- 

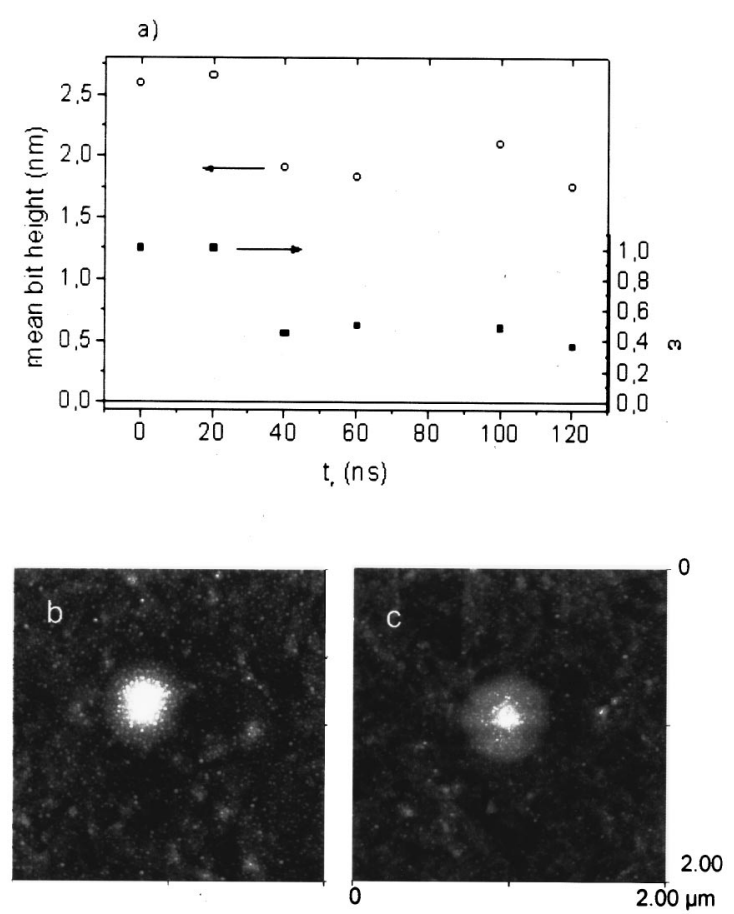

FIG. 8. (a) Remaining amorphous fraction $\epsilon$ for various erased bits in a hexagonal film as a function of the duration of the recrystallization pulse (closed squares). $\epsilon$ saturates at 0.4 , i.e., the original reflectance is not restored. The mean height of the same bits after the recrystallization pulse is also plotted (open circles). As for the fcc matrix a strong correlation to $\epsilon$ is found. (b)-(c) Partially and completely recrystallized bits. The amorphization parameters were $8.9 \mathrm{~mW}$ and $100 \mathrm{~ns}$. The power of the recrystallization pulse was $2.4 \mathrm{~mW}$. The duration of the pulse was 20 and $120 \mathrm{~ns}$, respectively. As for the fcc matrix, recrystallization leads to a decrease in bit height whereas the diameter of the bits remains unchanged.

flectance goes back to its original value $(\epsilon=0), \bar{h}$ reaches a saturation value of $\sim 2 \mathrm{~nm}$ due to the remaining coronal structure. This residual structure that comes from the erasure process is very similar to the structure formed during the melt crystallization process [cf., e.g., Figs. 6(h) and 7(f)]. So, completely erased bits still cause slight distortions of the surface topography which can be detected by the AFM.

An analogous examination of recrystallization was performed with amorphous bits in a hexagonal surrounding. The amorphization of the bits shown here in Sec. III B was performed with an incident laser power of $8.9 \mathrm{~mW}$. The length of the amorphization pulse was $100 \mathrm{~ns}$. The laser beam was again focused by an objective lens with $\mathrm{NA}=0.9$. As was also done earlier in Sec. III B, the power of the recrystallization pulse was held constant at $2.4 \mathrm{~mW}$ but the pulse duration was varied from 20 to 120 ns. Figure 8(a) reveals the same strong correlation between $\epsilon$ and $\bar{h}$ as for the fcc substrate. After $40 \mathrm{~ns}$ the same saturation height of $\sim 2 \mathrm{~nm}$ is attained. Here again the recrystallization expresses itself in a decrease of the center mound [Figs. 8(b) and 8(c)]. The coronal region remains unchanged and leads to a saturation value of $\bar{h}=2 \mathrm{~nm}$. But in contrast to the previous case (the fcc substrate) it is impossible to restore the original reflectance $(\epsilon=0)$. A saturation value of $\epsilon=0.45 \pm 0.05$ is obtained instead. This behavior can be explained assuming that the bits recrystallize into the fcc phase and not in the hexagonal phase of the substrate. As mentioned in Sec. II, the reflectance of the hexagonal phase is $10 \%$ higher than the reflectance of the fcc phase. Assuming that the recrystallization of the amorphous bit into the fcc phase is complete after $40 \mathrm{~ns}$, $R_{r}$ can then be written as $0.9 \times R_{c} . \epsilon$ can then be transformed into

$$
\epsilon=0.1\left(1-\frac{R_{a}}{R_{c}}\right)^{-1} .
$$

According to Eq. (3), $\epsilon$ depends only on $R_{a} / R_{c}$. The optical measurements lead to a mean value of $0.78 \pm 0.02$ for $R_{a} / R_{c}$. According to Eq. (3) this leads to $\epsilon$ of $0.46 \pm 0.06$. This is in excellent agreement with the measured value $(0.45 \pm 0.05)$. So, assuming that $R_{r}$ corresponds to the reflectance of the fcc phase, the measured value of $\epsilon$ can be reproduced. Moreover, evaluating $R_{c} / R_{r}$ for the completely recrystallized bits leads to $1.11 \pm 0.02$ which also corresponds very well to the $10 \%$ difference in reflectance between the hexagonal and fcc phases. This strongly supports the conclusion that erasure of amorphous bits leads to formation of the fcc phase even if the surrounding matrix consists of the hexagonal phase.

\section{DISCUSSION}

The results presented above provide ample evidence of the close correlation between the local changes of optical properties upon laser modification and the local topography of the bits produced. In particular, the topographic changes caused by local crystallization have been identified. Local crystallization leads to a volume reduction of the film since the surrounding amorphous matrix has a lower density. For a completely crystallized bit, a volume reduction of 6.0 $\pm 0.6 \%$ is observed by the AFM. This is in excellent agreement with the volume reduction of $6.2 \pm 0.8 \%$ found by $\mathrm{x}$-ray reflectometry measurements for films crystallized by heating in a furnace.

Previous AFM studies have reported either a strong $(40 \%)$ increase in film thickness ${ }^{18}$ or the formation of craters, which were several $10 \mathrm{~nm}$ deep, surrounded by an $\sim 100 \mathrm{~nm}$ high bulge ${ }^{19}$ upon laser irradiation of amorphous GeSbTe alloy films. In these studies the formation of bulges is attributed to the development of large crystal grains whereas the formation of the craters is explained by reamorphization upon melting. In light of the previous discussion this interpretation is not very likely. Instead, it appears as if the morphological changes reported are caused by local ablation. This is supported by a comparison with Fig. 3 which shows a sequence of areas modified with increasing laser power. While low power leads to crystallization [Fig. 3(a)], intermediate power leads to amorphization of the center spot [Fig. 3(b)]. A further increase of laser power leads to ablation of the center region and mound formation in the adjacent area [Fig. 3(c)]. Figure 3(c) closely resembles the structures presented in the AFM studies mentioned above. Hence it is very likely that ablation rather than crystallization has been described in these studies. Another interesting observation can be seen in Figs. 1(a) and 1(b). While crystalline bits that have been created by ns- $\mu$ s short pulses show cracks in the 
crystalline region, no such cracks are observed for crystalline bits produced by $\mu s-s$ long pulses. We attribute the difference to the time available to reduce local stresses. The 6.0 $\pm 0.6 \%$ volume reduction upon crystallization creates large stresses in the film. For long pulses these stresses can be reduced during laser heating by defect motion, such as dislocation glide. For short pulses the time available is insufficient to reduce stresses effectively. Hence the crystalline area remains under large stresses and forms cracks upon cooling. Amorphization is accompanied by a volume increase, which is attributed to the lower density of the amorphous phase. Recrystallization of amorphous bits leads to the formation of the fcc phase irrespective of the phase (fcc or hexagonal) of the surrounding material. Since the fcc and hexagonal phases possess different optical properties, it is not possible to achieve a complete erasure from the optical point of view $(\epsilon=0)$ when hexagonal films are used as the starting point. Complete erasure is only possible for fcc films. Even after complete recrystallization of the bit, there is still a slight $\sim 2$ $\mathrm{nm}$ high distortion of the surface topography which can easily be detected by the AFM [Fig. 7(d)].

The discussion presented above proves that the AFM is a versatile tool with which to examine bit topography, degree of crystallization, progress of recrystallization, etc. Yet only bits that were written in a phase change layer without an overcoat were imaged in this study. Due to the chemical reactivity, such uncoated films will not meet the stringent requirements for rewritable optical data storage. Therefore the question of whether similar studies can also be performed for phase change films with a protective overcoat arises. Since near-field recording on phase change media is particularly promising due to the very high storage density achievable, only thin overcoats should be considered. We have covered the $\mathrm{Ge}_{2} \mathrm{Sb}_{2} \mathrm{Te}_{5}$ film with a $10 \mathrm{~nm}$ thick $\mathrm{ZnS}-\mathrm{SiO}_{2}$ layer. These films were modified by the static tester and by individual bits subsequently imaged by the AFM. Examples of crystalline bits are shown in Fig. 9. The incident power was $2.3 \mathrm{~mW}$ and the pulse length $10 \mu \mathrm{s}$ [Fig. 9(a)] and $200 \mathrm{~ns}$ [Fig. 9(b)], respectively. This leads to a topography that is very close to that without an overcoat. This proves that analysis of bit topography and correlation with the change in optical reflectance are even possible for phase change films with an overcoat. Therefore the AFM is highly suitable tool that can improve the performance of phase change recording media.

\section{SUMMARY}

Access to the submicron length scale is crucial for the development of media with improved storage density and better signal-to-noise ratios. We have shown that the AFM is able to identify and characterize a variety of laser induced phase transitions in $\mathrm{Ge}_{2} \mathrm{Sb}_{2} \mathrm{Te}_{5}$ on a microscopic scale. This is possible since the amorphous and crystalline phases differ in density by $6 \%$. Therefore crystalline bits in an amorphous surrounding can be identified by the corresponding film depression, while amorphous bits in a crystalline matrix are identified by mound formation. As a consequence, writing and erasing of single bits can be studied. The depth of the
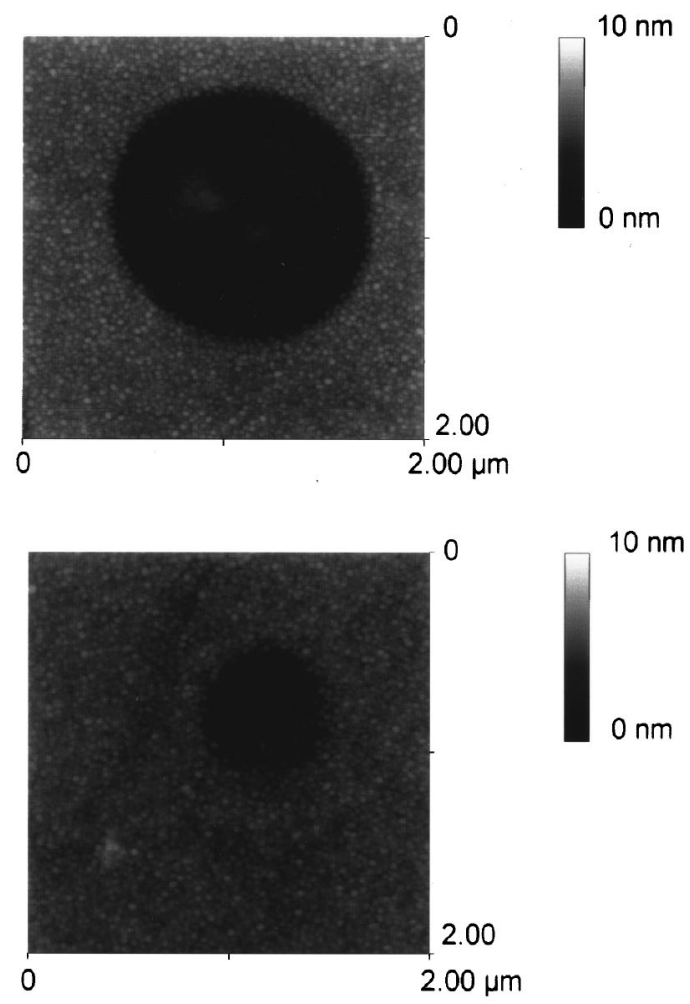

FIG. 9. AFM images of crystalline bits under a $10 \mathrm{~nm}$ thick $\mathrm{ZnS}-\mathrm{SiO}_{2}$ protective layer. The incident laser power was $2.3 \mathrm{~mW}$ and the duration of the pulse was (a) $10 \mu$ s and (b) $200 \mathrm{~ns}$, respectively.

depression reflects the degree of crystallization in the depressed region while the size corresponds to the crystallized area. Since the present setup allows the identification of individual bits by the AFM we are able to correlate the change of reflectance to the bit topography. This should help to pave the road to faster and better storage media with higher density.

\section{ACKNOWLEDGMENTS}

The authors thank J. Larscheid for technical support, P. Franz for XRR measurements, and H.-W. Woeltgens for optical reflectance measurements. Financial support by the Ministerium für Schule, Weiterbildung, Wissenschaft und Forschung des Landes Nordrhein-Westfalen, is gratefully acknowledged.

${ }^{1}$ K. A. Rubin, Mater. Res. Soc. Symp. Proc. 230, 239 (1992)

${ }^{2}$ B. A. J. Jacobs and J. P. W. B. Duchateau, Jpn. J. Appl. Phys., Part 1 36, 491 (1997).

${ }^{3}$ N. Yamada, Proceedings of the ODS Topical Meeting, Tucson, April 1997

${ }^{4}$ T. Ohta, K. Nagata, I. Satoh, and R. Imanska, IEEE Trans. Magn. 34, 426 (1998).

${ }^{5}$ I. Satoh, S. Ohara, N. Akahira, and M. Takenaga, IEEE Trans. Magn. 34, 337 (1998).

${ }^{6} \mathrm{M}$. Libera and M. Chen, MRS Bull. 1990, 40.

${ }^{7}$ N. Yamada, MRS Bull. 1996, 48.

${ }^{8}$ E. Betzig and J. K. Trautman, Science 257, 189 (1992).

${ }^{9}$ B. D. Terris, H. J. Mamin, D. Rugar, W. R. Studenmund, and G. S. Kino, Appl. Phys. Lett. 65, 388 (1994).

${ }^{10}$ S. Hosaka, T. Shintani, M. Miyamoto, A. Hirotsune, M. Terao, M. Yoshida, K. Fujita, and S. Kämmer, Jpn. J. Appl. Phys., Part 1 35, 443 (1996). 
${ }^{11}$ T. Suzuki, Y. Itoh, M. Birukawa, and W. Van Drent, IEEE Trans. Magn. 34, 399 (1998).

${ }^{12}$ J. Tominaga, H. Fuji, A. Sato, T. Nakano, T. Fukaya, and N. Atoda, Jpn. J. Appl. Phys., Part 2 37, L1323 (1998).

${ }^{13}$ I. Friedrich, V. Weidenhof, P. Franz, W. Njoroge, and M. Wuttig, J. Appl. Phys. (submitted).
${ }^{14}$ H.-W. Woeltgens, I. Friedrich, W. Njoroge, and M. Wuttig (unpublished).

${ }^{15}$ B. Lengeler and H. Hüppauff, Fresenius J. Anal. Chem. 346, 155 (1993).

${ }^{16}$ H. Zabel, Appl. Phys. A: Solids Surf. 58, 159 (1994).

${ }^{17}$ C. Volkert and M. Wuttig, J. Appl. Phys. 86, 1808 (1999).

${ }^{18}$ Z. Wu, F. Gan, J. Hu, and M. Li, Appl. Surf. Sci. 72, 245 (1993).

${ }^{19}$ L. Men, F. Gan, J. Sun, and M. Li, Appl. Surf. Sci. 120, 171 (1997). 